\title{
Handling machine breakdown for dynamic scheduling by a colony of cognitive agents in a holonic manufacturing framework
}

\author{
T. K. Jana ${ }^{\text {a }}$, S. Naskar ${ }^{\mathrm{a}}$, S. Paul ${ }^{\mathrm{b}}$, B. Sarkar ${ }^{\mathrm{c}}$ and J. Saha ${ }^{\mathrm{c}}$
}

${ }^{a}$ Department of Mechanical Engineering, Haldia Institute of Technology, Haldia 721 657, India ${ }^{b}$ Department of Information Technology, Haldia Institute of Technology, Haldia, 721 657, India ${ }^{c}$ Department of Production Engineering, Jadavpur University, Kolkata 700 032, India

\section{H R O N I C L E}

\begin{tabular}{l}
\hline Article history: \\
Received March 29, 2015 \\
Received in revised format: \\
May 12, 2015 \\
Accepted May 22, 2015 \\
Available online \\
May 222015 \\
\hline Keywords: \\
Cognitive \\
Contract net protocol \\
Distributed scheduling \\
Multi agent based holonic \\
adaptive scheduling \\
Metamorphosis \\
Self-organizing
\end{tabular}

\section{Introduction}

The success of a production system, by and large, is governed by the shop floor control activities and resource scheduling is one of the most prominent issues to be addressed in this regard. Traditional centralized scheduling although provides optimal or near optimal solution by using varieties of powerful enumerative algorithms and is prepared well in advance, it often becomes of little significance during time of implementation, since shop floor condition changes abruptly from what it was envisaged (Shen, 2002; Leitao \& Restivo, 2008). This is owing to the fact that such schedules are less responsive

\footnotetext{
* Corresponding author. Tel.: +91 3224252900 (Extn. 258); Fax: +91 3224252800

E-mail address: tarun.jana2000@gmail.com (T. K. Jana) 
to changes and hence inflexible and therefore, unable to satisfy the unexpected dynamic shop floor requirements (Brussel et al., 1999; Shen, et al., 2006a; Leitao \& Restivo, 2008; Lou et al., 2010; Wang et al., 2012). This essentially calls for dynamic scheduling so that schedule can be generated depending on the real-time information.

The problems of centralized scheduling can be overcome to a great extent by adopting decentralized approach where decision making is delegated to the individual entities for scheduling, giving rise to the concept of distributed scheduling (DS). DS is an approach, derived from the concept of distributed problem solving (DPS) (Smith, 1980; Smith \& Davis, 1981), enables the local decision makers to prepare schedules by considering local objectives and constraints within the boundaries of the overall system objectives (Toptal \& Sabuncuoglu, 2010). In this paradigm, the local decisions are made quickly using the most recent system information, and therefore overall schedule is more responsive to dynamic and unpredictable events, such as machine breakdowns, new job arrivals, or order cancellations. Local decisions of the individual entities are then integrated through coordination and communication mechanisms to create the complete global schedule. Since distributed scheduling problems usually attempt to achieve only its local objective without considering the global objective, a contradictory problem might occur between the local objective and the overall system performance (Chan \& Chung, 2013). The aim of distributed scheduling is to enhance system flexibility, agility, and utilization of resources through effective allocation of processes (tasks) by collaboration between system entities. Additionally, it offers robustness to the scheduling under the changing circumstances. Owing to its dynamic nature, combinatorial aspects, and its practical interest, the distributed scheduling problem has gained substantial attentions in the literature (Lou et al., 2010; Toptal \& Sabuncuoglu, 2010; Chan \& Chung, 2013).

The multi-agent based holonic manufacturing control has been considered as a very promising platform to execute distributed scheduling in dynamic manufacturing environments (Shen, 2002; Walker et al., 2005; Shen et al., 2006a, Shen et al., 2006b; Wong et al., 2006a, 2006b; Leitao \& Restivo, 2008; Cao et al., 2009; Lou et al., 2010, Toptal \& Sabuncuoglu, 2010; Renna, 2011; Chan \& Chung, 2013). The agent-based approach benefits the manufacturing scheduling in various ways, the most prominent being the integration of process planning and scheduling (IPPS) functions. IPPS facilitates information exchange in real-time to make the planning and scheduling a realistic one and also to optimize both simultaneously as a single problem, considering the constraints of both domains (Tan \& Khoshnevis, 2000; Shen et al., 2006b; Wong et al., 2006b; Wang et al., 2006; Cai et al., 2009; Li et al., 2010; Phanden et al. 2011).

The behavior of an agent has a close resemblance to that of a human being as reflected in societal systems (Wooldridge \& Jennings, 1995; Monostori et al., 2006; Leitao, 2009; Meyer et al. 2009). Agents exhibit two contradictory properties: under normal situation, they are highly competitive and vie with each other for lucrative jobs to augment their earnings and credentials, but when disturbances try to cripple the system, agents resort to cooperation (Jana et al. 2013). Under an abnormal situation, agents metamorphose (Wang et al., 1998; Balasubramanian et al. 2001) into the new environments so as to retain their sangfroid by providing solution and to get rid of the crisis.

Disturbance handling is one of the major issues in scheduling and agent based approach is very efficient to cope with such situations. Disturbances refer to random occurrence of events that prohibit achieving the goal and causes perturbations to the system such as rush order, cancellation/modifications of order quantity, machine partial and complete malfunctioning, changes in delivery pattern and priority, addition/alteration of resources, non-conformance to quality requirements etc. (Vieira et al., 2003; Wong et al., 2006b; Leitao \& Restivo, 2008).

A detailed study on rescheduling under disturbances is reported in the literatures (Vieira et al., 2003; Ouelhadj \& Petrovic, 2009). It emerges from their study that in the event of machine failure, the 
completion time of the affected job and the other subsequent jobs would be delayed by an amount equals to the down time. However, the deviation or lag can be reduced by incorporating some slack to enhance the robustness of the schedule predictability. Disturbance handling due to machine malfunctioning by agent based holonic approach is credited to several researchers (Bongaerts et al. 1997; Wong et al., 2006b; Wang et al., 2008; Leitao \& Restivo, 2008; Hsieh, 2010; Nejad et al., 2011; Leitao, 2011). It follows from these literatures that in the wake of such situations, rescheduling is required and a delay is on the cards. This rescheduling is carried out by renegotiation, which is always accompanied by undesirable ripple effects (Gao et al., 2005) that adversely affect system's performance (Wang et al., 2012). Further, optimization for rescheduling requires extra procedures, which is often accomplished by higher authority (Bongaerts et al., 1997; Leitao \& Restivo, 2008). Additionally, no attempt is paid to reduce the delay by expediting the incomplete work and other remaining jobs, if possible.

The conventional automated production systems are therefore unable to deal with growing shop floor complexities. Human workers by virtue of their natural cognitive capabilities offer high reactivity, agility, and adaptability and hence can deal with such a traumatic situation (Park \& Tran, 2012). It is therefore need of the hour that modern production systems should be harness with the cognitive principles to execute planning and control of production systems to meet the challenges (Nobre et al., 2008; Bannat et al., 2011). The self-organizing (Tharumarajah, 2003; Serugendo et al., 2006; Leitao et al., 2012) ability of the cognitive agents, which is the manifestation of proactiveness, helps to form a coalition in response to the environmental changes so as to provide solution under abnormal condition.

To deal with the machine breakdown, we propose a novel self-organizing mechanism of the cognitive agents, which is imitated from the behavior of human being, to reallocate the incomplete job in the most optimized manner. Once a resource confronts any malfunctioning, a triggering mechanism fires implicitly to initiate a course of actions that eventually determine the earliest possible completion time of the affected job by different active resources that would be considered as criterion of task reallocation. Furthermore, under such situations, these resources always opt for the optimum process plan for subsequent processing so as to expedite the execution in an attempt to minimize the makespan and the delay.

The present work is focused at the fault tolerant machine scheduling by a group of cognitive agents in a holonic manufacturing framework. The work is essentially an augmentation of our earlier work (Jana et al., 2014) in regard to achieving improved performance under disturbance by cognitive behavior of the agents. The objective is to justify the credentials that cognitive agents perform better than the ordinary agents under uncertainty. Initially, an explicit communication based negotiation procedure is adopted to accomplish multi objective scheduling under normal situation. When breakdown of a resource takes place, the task reallocation is accomplished by implicit sensing and coordination. Based on the proactivness of the cognitive agents, a metamorphic algorithm is developed that can identify the best candidate resource to whom the incomplete task would be offered. Additionally, metamorphosis helps the active resources to accelerate the work so as to nullify the adverse consequences of machine breakdown as far as possible.

The rest of the paper is organized in the following ways. The concept of cognitive agents and cognitive decision making is presented in Section 2. The negotiation based scheduling under normal situation is presented in Section 3. Section 4 deals with the schedule modifications under disturbance by an implicit coordinated approach. Implementation of the proposed scheduling technique is presented in Section 5. Results and performances are discussed in Section 6. Finally, Section 7 concludes the paper. 


\section{Cognitive agent}

Cognitive agents are software entities that can imitate human being in regard to intelligent decisionmaking by performing three-step activities namely perception, reasoning, and execution (Zhao \& Son, 2008). These agents also possess the basic characteristics of traditional agents such as autonomy, social ability, and reactivity. The cognitive agents are being fortified with higher level decision-making ability, offer enhanced flexibility and manifest better adaptability under changing situations. A simple cognitive decision making scheme by human being is shown in Fig. 1. The functionality relies on Beliefs-Desires-Intentions (BDI) model (Rao \& Georgeff 1998; Lee \& Son 2008). Beliefs are the facts about the environment. In the context of the manufacturing shop-floor, it implies the recent and updated information about the status of the machines and the processes. Desires are the goals or end states which an agent wants to attain, and the intentions refer to an agent's commitments to its desires (goals) and adherence to the plans or strategies to achieve those goals. The sensing module collects the information from the environment. The cognitive planning and control module interprets the acquired information by reasoning and accordingly generates the action plans based on knowledge base. Additionally, a communication module establishes to and fro communication among the other members of the entity.

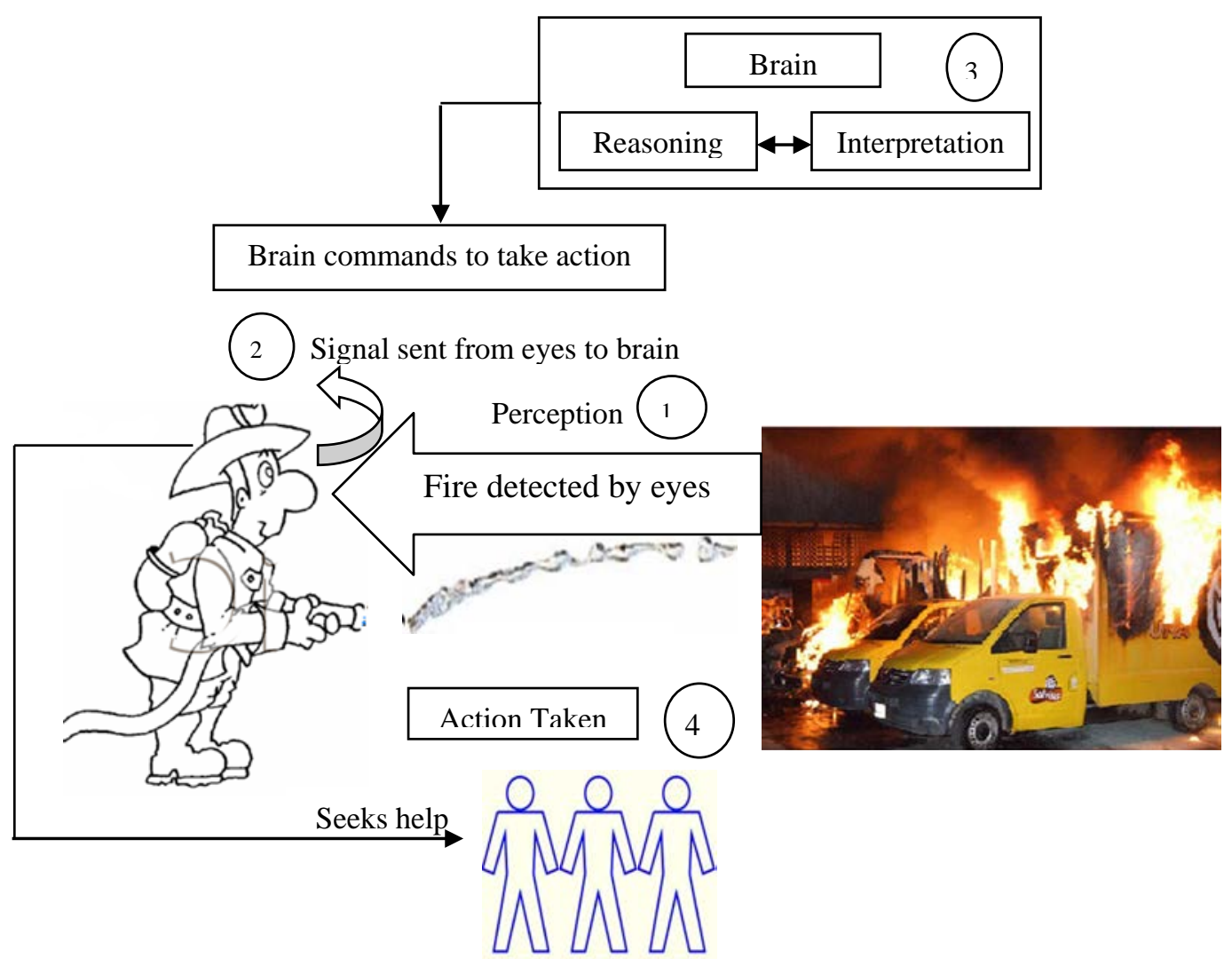

Fig. 1. A cognitive decision making model by human being

\section{Negotiation based scheduling}

The negotiation based scheduling procedure remains same as reported in our earlier work (Jana et al. 2014) under normal situation. The products get the opportunity for negotiation with the resources according to the rank, settle the agreement by negotiation, and prepare the local schedules. The schedule generated under normal condition would act as reference only for performance comparison with those under disturbances. 


\subsection{The System Architecture}

The proposed holonic system architecture, named Multi Agent based Holonic Adaptive Scheduling (MAHoAS), is presented in Fig. 2. It reflects the combination of hierarchical and heterarchical system, leading to a hybrid one. At the top of the hierarchy, there is system supervisor. Any order for execution is decided by him and accordingly the product holon is created. The system supervisor computes the revenue that can be generated by processing a product, establishes its due date (relative urgency), and passes these information to the integrated process planning \& scheduling holon for product ranking. Additionally, the supervisor affixes the deadline based on virtual scheduling (see Section 3.2) and intimates it to the products as well as to the resources. The integrated process planning \& scheduling holon, located at the intermediate level, generates the operation plan and detailed process plan for the products and resources respectively. Additionally, this holon develops the priority of the products, and frames the schedule guidelines. The product holon and the resource holon, both located at the bottom level of the hierarchy, negotiates for task allocation. Further, resources can interact with each other to achieve cooperation based work.

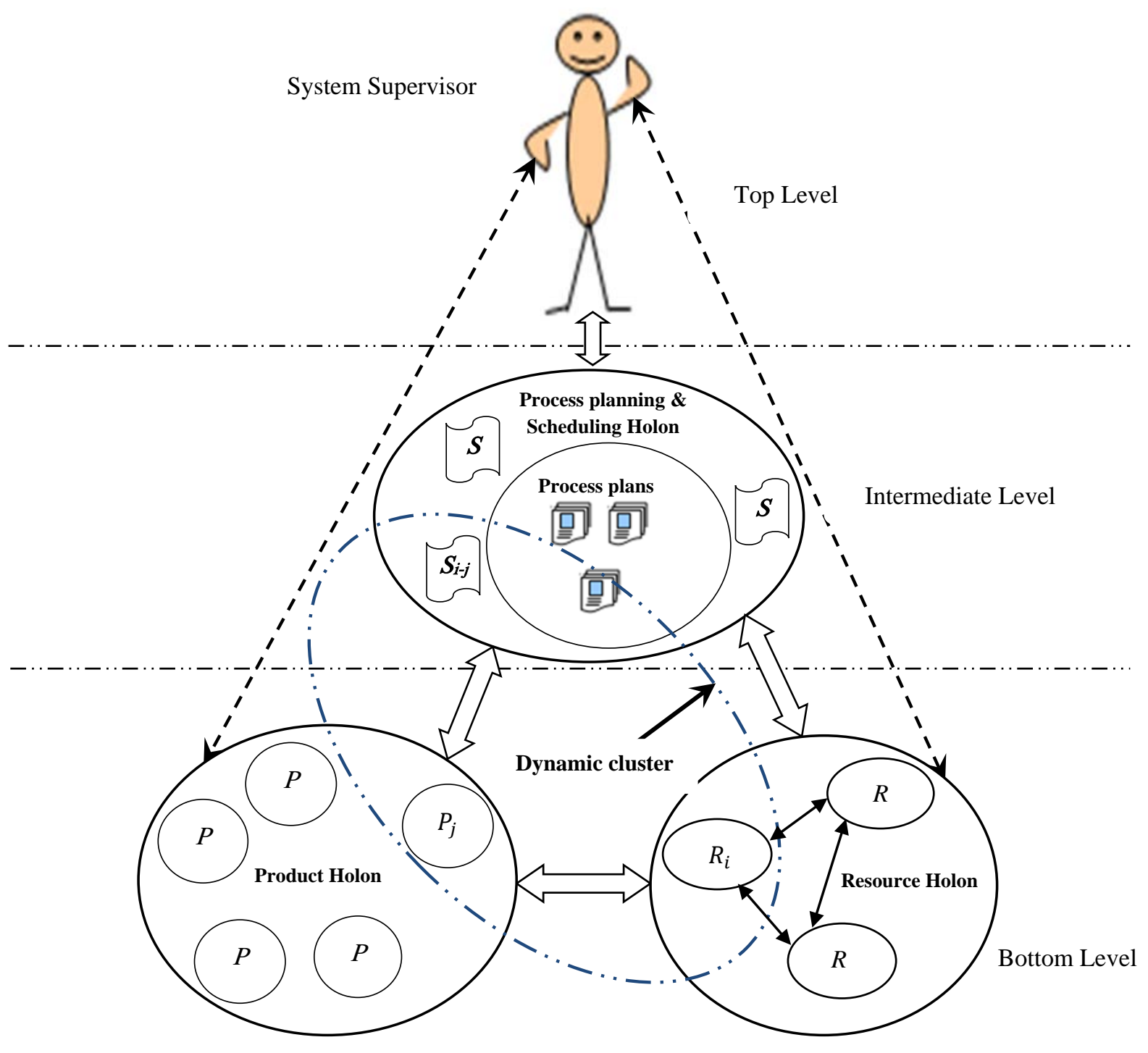

Fig. 2. Multi Agent based Holonic Adaptive Scheduling (MAHoAS) architecture

The products and the resources are also permitted to interact with the supervisor. This helps to realize the bottom-up approach. The presence of system supervisor ensures hierarchy in the system only to be 
maintained in loosely coupled manner by establishing congruence among the different agent communities. However, when disturbances are present, the system migrates from hierarchical control to what is called heterarchical control to manifest more agility so as to confront the challenges by adapting to new situations. Resources also transform from hitherto competitive attitude to a cooperative attitude to solve the problem collectively. The communication among the system entities is envisaged to be established by client-server model using J2EE technology (Jana et al. 2013).

\subsection{Schedule preparation and establishing deadline}

The scheduling approach followed in the current research falls under the category of pure reactive scheduling (Ouelhadj \& Petrovic, 2009) i. e. no schedule is generated beforehand. The products and the resources, by virtue of autonomy, prepare their own schedules (the same schedule rule is followed as reported in Jana et al. 2014.) dynamically by negotiation considering the recent information and fit into the time window. However, a virtual schedule for all the products is completed before commencement of actual negotiation. Virtual schedule implies how the negotiation based schedules would take the shape, if everything goes right under normal condition (in the absence of any disturbance). This prima-facie complete global picture of the schedule under normal condition would facilitate the system supervisor to see the earliest possible completion time (make span) and to affix the deadline. System supervisor establishes a deadline following forward scheduling approach considering the longest time taken by any resource and an allowance of $12.5 \%$ to take care of any deviation. This deadline is intimated to the resources so that the products can be completed within this time frame. It is noteworthy that actual schedule is liable to change from the virtual one in the event of disturbances. Once this deadline is communicated to the resources, actual negotiation is initiated and execution commences.

\section{Schedule modifications under disturbances}

When a particular resource malfunctions and breaks down completely, it tries to recover at the earliest. If it is diagnosed that recovery is not possible within a short while, the faulty resource communicates with others seeking help to take the onus of incomplete work. Since the number of resources is reduced, there is a fair likelihood of not meeting the deadline. However, success or failure to meet the deadline depends on how much progress is already been made at the time of failure and to what extent the processing of the remaining work of the incomplete job and the other products (those are waiting in the queue for processing) can be expedited.

\subsection{Metamorphosis}

Like human being, a cognitive agent has two facets of attitude towards work: (i) reactivity - implying the ability of any agent to respond and execute an instruction, and (ii) proactivity - that manifests its ability to initiate a work from its own as being opposed to the previous one. The former being more prominent in a static and stable environment, the later one plays crucial role in dynamic situations when disturbances prevail. Nevertheless, both these attributes are present simultaneously in varying amount in any intelligent system. However, their proportion at any particular instance is a trade-off between the two. An agent imbibes proactiveness that dictates how it responds to any disturbance by virtue of metamorphosis.

\subsection{The Philosophy of cooperation}

The cooperation based work, which is the manifestation of self-organizing behavior of an agent, is characterized by the following premises. 
(i) The customer requirements (in time completion of the tasks) would be given paramount importance than the interests of the individuals (augmentation of individual credentials, more earnings etc.),

(ii) It is postulated that the successful completion of tasks eventually brings glory to the team as a whole rather than individual and failure, on the other, is ignominious to the entire community,

(iii) There would be an all out effort by the resources to overcome the disturbances.

\subsection{The motivation behind cooperation}

The initiative to shoulder extra work is motivated by some earnings. When any resource fails to complete its stipulated work due to malfunctioning and seeks help from others, the resource that eventually provides help, deserves a payment for rendering services. It is reasonable to consider that the resource which takes the responsibility of processing the incomplete work of the faulty resource should get payment commensurable to the work content [which is not done by the faulty resource] in addition to some incentives as stimulant and this entire amount would be paid by the faulty resource.

Say a particular resource $R_{k}$ breaks down while processing the product $P_{l}$ and therefore it remains incomplete by $R_{k} \cdot R_{i}$ is an active resource (where $i=1,2, . ., m, i \neq k$ ), that provides help in this regard. Thus earnings $[E]_{R_{i}}^{P_{l}}$ of $R_{i}$ that executes the incomplete work of $P_{l}$ is modeled as

$$
[E]_{R_{i}}^{P_{l}}=(1-\alpha) \cdot[\sigma]_{R_{k}}^{P_{l}}+I
$$

where, $[\sigma]_{R_{k}}^{P_{l}}$ is the payment supposed to be paid by the faulty resource $R_{k}$ for complete execution of product $P_{l}, \alpha$ is the fraction of the work already executed by $R_{k}$ at the time of failure, and $I$ is the incentive amount as mutually agreed and paid by $R_{k}$.

\subsection{Cooperation mechanism by cognitive agents}

Inspired by human-decision making model, a cognitive architecture, derived from the cognitive science and based on BDI model, is developed and presented in Fig. 3 to realize cooperation based work by a colony of cognitive agents (resources). When breakdown of any resource takes place, the colony of resources forms a network of implicit communication, which is inspired by nature. The explicit communication for renegotiation seems inappropriate under this changing circumstance since such practice is coupled with several disadvantages (Gao et al., 2005; Wang \& Tang, 2011; Wang et al., 2012). The coordinated decision making by implicit communication is a behaviour predominant in colonies of insects, known as swarm intelligence (Nanvala \& Awari, 2011; Leitao et al., 2012), is an efficient approach to deal with the crisis situation arising out of machine malfunctioning. The problem solving approach by the colonies of insects can be viewed as distributed and parallel systems without the presence of any hierarchy (Hirsh \& Gordon, 2001). Ant colony optimization (ACO) (Dorigo et al., 1996; Li et al., 2011) and Particle swarm optimization (PSO) (Zhao et al., 2007; 2011) are two predominantly used algorithms under this paradigm to optimize global system performances.

In our proposed system, once any resource confronts a malfunctioning, the situation is intimated to the other resources in the group by an alarm signal through the communication network system using J2EE technology. This action is analogous to the pheromone deposition by the ant colony to propagate any information to the community that relies on the principle of stigmergy (Hadeli et al., 2004; Gao et al., 2005; Valckenaers et al., 2007; Wang \& Tang, 2011; Wang et al., 2012). However, unlike evaporation of pheromone, this alarm signal remains active until the resource recovers from fault and resumes its work. Consequently, the progress made at the time of failure is also communicated to other resources to evaluate the pending work content and to determine the associated time required to complete it. Message of failure of any resource invigorates the other resources in the group to shoulder the 
responsibility of finishing the incomplete work of defective resource and to complete the entire product batch at the earliest. The fault message acts as a trigger and fires implicitly to undertake a series of computations to determine the earliest completion time of the affected product.

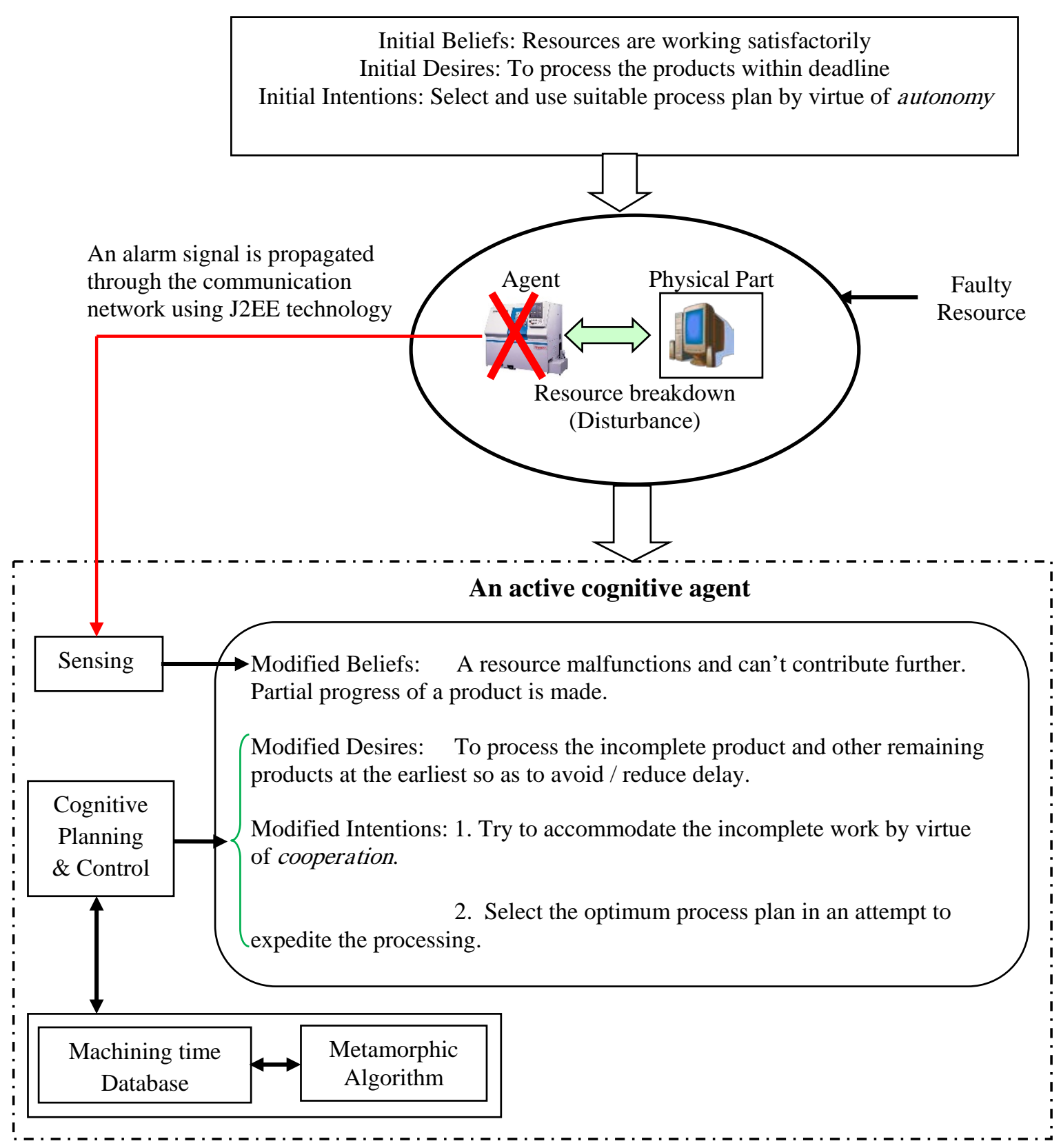

Fig. 4. The proposed cognitive architecture for handling machine breakdown

\subsection{The cooperation strategy}

The cooperation strategy adopted under this scenario relies on the proactive behaviour of the cognitive agents. A course of actions to tackle this situation is pre-formulated. During negotiation, the cognitive agents consider multiple process plans and select any particular plan that seems befitting for bidding under normal situation. However, the multiple process plans serve several purposes of the agent community as follows.

(a) In view of failure of the current plan (say breakage of a cutting tool), the resource can switch over to another plan, thereby enhancing the flexibility. 
(b) When break down of any resource takes place in such a way that recovery is not possible within short time, then the incomplete work would be shouldered by other agents of the colony. Under such situation, reduction of number of resources would tend to delay the completion. Nevertheless, the remaining active resources try to expedite the rest of their current work by following different (optimum) process plan, if available, to complete it at the earliest and try to accommodate the incomplete work as soon as possible to reduce the delay [refer Fig. 3]. Again, while dealing with the partially processed work, a resource would also select a process plan which incurs minimum time, and (c) In an attempt to compensate the lag and to reduce the make span, the resources always select the best plan and incorporates the corresponding machining time in the bid during subsequent negotiation.

A metamorphic algorithm is developed that determines the expected early finishing time of the incomplete work by various active resources. The algorithm is presented by a flow chart in Fig. 4 .

Following symbols are used in the algorithm

$$
\begin{gathered}
{\left[T_{b}\right]_{R_{k}}^{P_{l}}} \\
{\left[T_{s}\right]_{R_{k}}^{P_{l}}} \\
{\left[T_{s}\right]_{R_{i}}^{P_{j}}} \\
{\left[T_{c}\right]_{R_{i}}^{P_{j}}} \\
{\left[\tau_{o p}\right]_{R_{k}}^{P_{l}}} \\
{\left[\tau_{o p}\right]_{R_{i}}^{P_{j}}}
\end{gathered}
$$$$
\left[\left(\tau_{\text {op }}\right)_{\min }\right]_{R_{i}}^{P_{l}}
$$$$
\left[\left(\tau_{\text {op }}\right)_{\min }\right]_{R_{i}}^{P_{j}}
$$$$
\left[\left(\tau_{\text {reqd }}\right)_{\min }\right]_{R_{i}}^{P_{l} / R_{k}}
$$

$\alpha$

$\beta$

$$
\left[\left(\tau_{\text {reqd }}\right)_{\min }\right]_{R_{i}}^{P_{j}}
$$

time of breakdown of $R_{k}$ while processing $P_{l}$

starting time of $P_{l}$ by $R_{k}$

starting time of $P_{j}$ by $R_{i}$ where $i=1,2, . ., m, i \neq k$ and $j=1,2, . ., n, j \neq l$

completion time of $P_{j}$ by $R_{i}$

stipulated processing time of $P_{l}$ by $R_{k}$ corresponds to the process plan currently followed

stipulated processing time of $P_{j}$ by $R_{i}$ corresponds to the process plan currently followed

minimum processing time of $P_{l}$ by $R_{i}$ following the optimum process plan

minimum processing time of $P_{j}$ by $R_{i}$ following the optimum process plan

minimum time required to complete the balance work of $P_{l}$ (which was partially executed by $R_{k}$ ) by $R_{i}$

fractional progress of $P_{l}$ by $R_{k}$ at the time of breakdown of $R_{k}$ and computed as $\alpha=\frac{\left[T_{b}\right]_{R_{k}}^{P_{l}}-\left[T_{s}\right]_{R_{k}}^{P_{l}}}{\left[\tau_{\text {op }}\right]_{R_{k}}^{P_{l}}}$

fractional progress of $P_{j}$ by $R_{i}$ at the time of breakdown of $R_{k}$ and computed as $\beta=\frac{\left[T_{b}\right]_{R_{k}}^{P_{l}}-\left[T_{s}\right]_{R_{i}}^{P_{j}}}{\left[\tau_{o p}\right]_{R_{i}}^{P_{j}}}$

minimum time required to complete $P_{j}$ by $R_{i}$ from the instance of occurrence of breakdown of $R_{k}$ following the optimum process plan 
$\left[E F T_{\exp }\right]_{R_{i}}^{P_{l} / R_{k}}$ of $P_{l}$ (partially time span to finish $P_{l}$ from the instance of occurrence of breakdown of processed by faulty $R_{k}$ resource $R_{k}$ before failure)

by a capable resource $R_{i}$

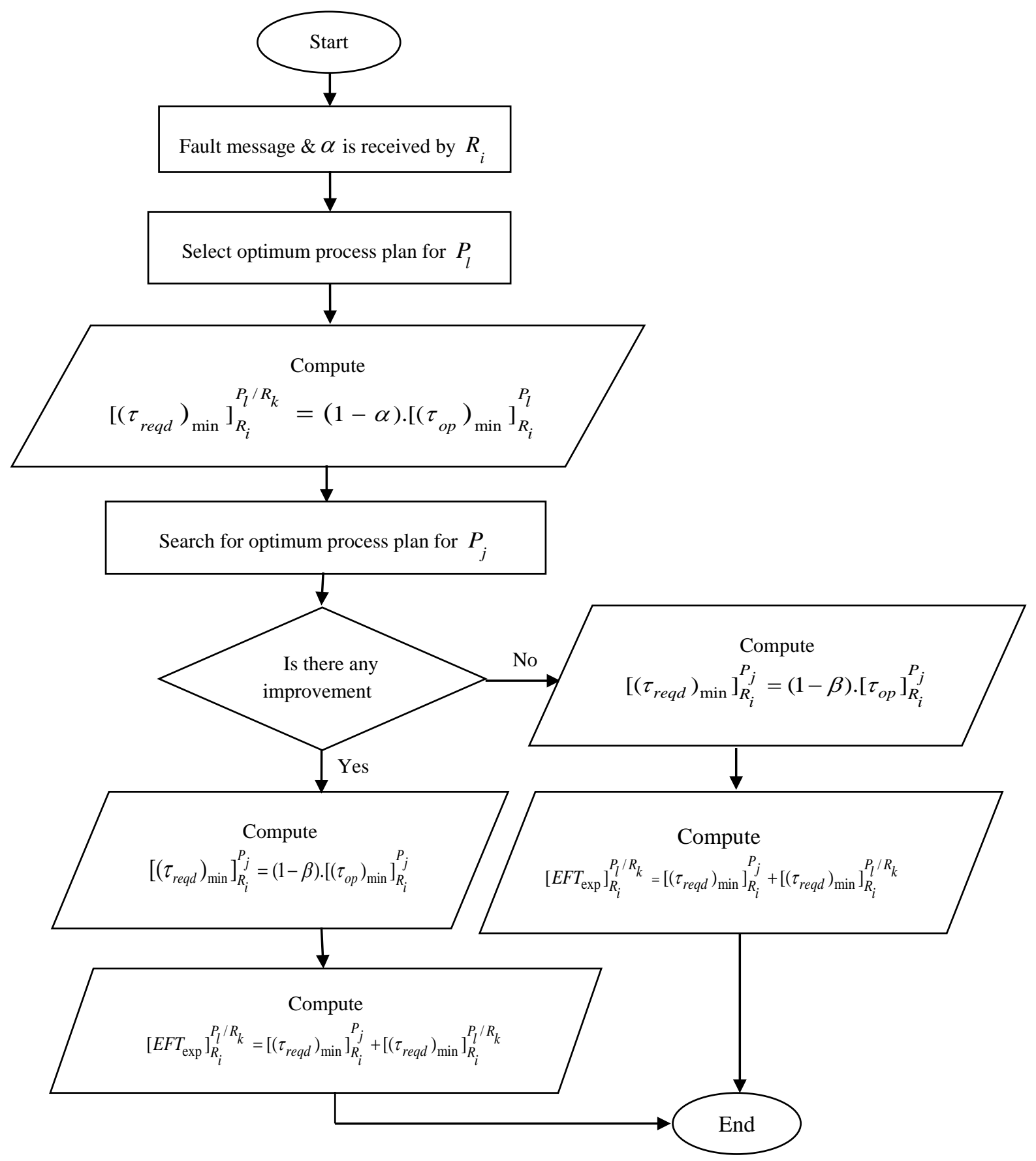

Fig. 4. Flowchart showing execution of the metamorphic algorithm

The above algorithm is implemented as a trigger to undertake a set of actions. The EFT of the incomplete product by various active resources is sent to the faulty resource and the later offers the task to one having the minimum EFT. Once the incomplete task is re-allocated, the original schedule rule is enforced once again for those products waiting in the queue for negotiation. 
The proposed coordination and cooperation mechanism together with the original schedule rule is advantageous in many respects. (i) The metamorphic algorithm is inspired by nature and therefore simple, yet comprehensive. Further, the algorithm attempts to expedite the processing by adopting optimum process plan, (ii) it optimizes the task re-allocation, (iii) since the schedule preparation is based on the real-time information, no advance schedule is generated and therefore the question of rescheduling arises only for the affected job, (iv) implicit cooperation and coordination mechanism does not call for any renegotiation and hence free from its negative consequences, (v) after reallocation of the incomplete job, the original schedule rule is followed that eventually ensures automatic load balancing even under changing circumstances.

\section{Implementation}

To investigate the performance of cognitive agents in comparison to the ordinary agents, we consider the same platform (Jana et al., 2014) having a resource holon comprising three resources (Turning centers) ( $\mathrm{R}_{1}$ to $\mathrm{R}_{3}$ ) and a product holon having eight products ( $\mathrm{P}_{1}$ to $\left.\mathrm{P}_{8}\right)$. Additionally, the times of failure of the resources are also considered unchanged. The challenge is how the incomplete work of the faulty resource would be re-allocated in the most efficient manner and how the resources can expedite the subsequent operations so as to minimize the ill-effects of the resource failure. To compare the scheduling performance under disturbances, few case studies are conducted and the corresponding scheduling results are compared with the normal one to evaluate the effects of disturbance. The schedule under normal situation is considered for reference only and presented in Fig. 5.

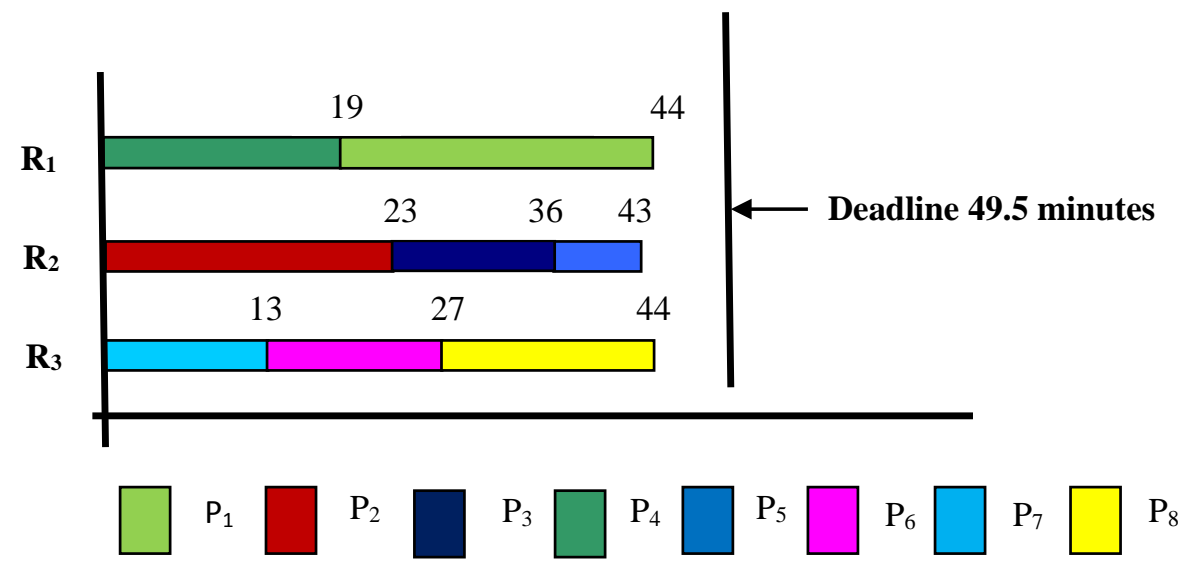

Fig. 5. Gantt chart showing schedule under (N)

The machining time database for various product-resource combinations using multiple process plans are presented in Table 1. The bold faced values of Table 1 are considered for bidding under normal situation $(\mathrm{N})$ and these are not necessarily the best ones.

\section{Table 1}

Database of machining time of various product-resource combinations using multiple process plans

\begin{tabular}{|c|c|c|c|c|c|c|c|c|}
\hline Resource & $P_{1}$ & $P_{2}$ & $\mathrm{P}_{3}$ & $\mathrm{P}_{4}$ & $P_{5}$ & $\mathrm{P}_{6}$ & $P_{7}$ & $\mathrm{P}_{8}$ \\
\hline \multirow{3}{*}{$\mathrm{R}_{1}$} & $\mathrm{t}_{111}=23.0$ & $t_{121}=18.0$ & $\mathrm{t}_{131}=11.0$ & $\mathrm{t}_{141}=19.0$ & $\mathrm{t}_{151}=04.0$ & $\mathrm{t}_{161}=09.0$ & $t_{171}=08.0$ & $t_{181}=12.0$ \\
\hline & $t_{112}=25.0$ & $\mathrm{t}_{122}=20.0$ & $t_{132}=12.0$ & $\mathrm{t}_{142}=20.0$ & $t_{152}=04.5$ & $t_{162}=10.0$ & $t_{172}=09.0$ & $\mathrm{t}_{182}=13.5$ \\
\hline & $\mathrm{t}_{113}=26.0$ & $\mathrm{t}_{123}=21.0$ & $\mathrm{t}_{133}=12.5$ & & $t_{153}=05.0$ & $\mathrm{t}_{163}=11.0$ & $\mathrm{t}_{173}=10.0$ & $\mathrm{t}_{183}=14.0$ \\
\hline \multirow{3}{*}{$\mathrm{R}_{2}$} & $\mathrm{t}_{211}=29.0$ & $\mathrm{t}_{221}=22.0$ & $\mathrm{t}_{231}=15.0$ & $t_{241}=21.0$ & $\mathrm{t}_{251}=7.50$ & $t_{261}=12.0$ & $t_{271}=11.0$ & $t_{281}=15.0$ \\
\hline & $t_{212}=31.0$ & $t_{222}=23.0$ & $\mathrm{t}_{232}=14.0$ & $\mathrm{t}_{242}=23.0$ & $t_{252}=07.0$ & $\mathrm{t}_{262}=13.5$ & $\mathrm{t}_{272}=12.0$ & $t_{282}=16.0$ \\
\hline & $t_{213}=34.0$ & $\mathrm{t}_{223}=26.0$ & $t_{233}=13.0$ & $\mathrm{t}_{243}=23.5$ & $\mathrm{t}_{253}=7.30$ & $202 . .$. & 2. & $t_{283}=16.5$ \\
\hline \multirow{3}{*}{$\mathrm{R}_{3}$} & $t_{311}=37.0$ & $t_{321}=28.0$ & $\mathrm{t}_{331}=17.0$ & $t_{341}=24.0$ & $t_{351}=08.0$ & $t_{361}=14.0$ & $\mathbf{t}_{371}=13.0$ & $t_{381}=17.0$ \\
\hline & $\mathrm{t}_{312}=41.0$ & $\mathrm{t}_{322}=29.0$ & $\mathrm{t}_{332}=19.0$ & $\mathrm{t}_{342}=25.0$ & $\mathrm{t}_{352}=10.0$ & $\mathrm{t}_{362}=15.5$ & $\mathrm{t}_{372}=15.0$ & $t_{382}=19.0$ \\
\hline & ........ & $\mathrm{t}_{323}=32.0$ & $t_{333}=16.0$ & $\mathrm{t}_{343}=27.0$ & $\mathrm{t}_{353}=12.0$ & ........ & ........ & $\mathrm{t}_{383}=21.0$ \\
\hline
\end{tabular}

Note: The numerical values appeared in the suffices imply the order resource - product - process plan number 
It is the sole prerogative of the resources to consider a particular plan that seems appropriate for bidding. The deadline is estimated as 49.5 minutes ( 44 minutes $+12.5 \%$ allowances), considering $\mathrm{R}_{1}$ and $\mathrm{R}_{3}$ as the critical. Three different bottleneck scenarios (D1 - D3) are considered for investigation. The proposed metamorphic algorithm is implemented in Oracle 9i. The break down details and the procedural outcome of the algorithm for the scenarios (D1 - D3) is presented in Table 2.

\section{Table 2}

Details of resource break down scenario and procedural outcome of metamorphic algorithm

\begin{tabular}{|c|c|c|c|c|c|}
\hline Scenario & $R_{k}$ & $P_{l}$ & $\begin{array}{c}{\left[t_{b}\right]_{R_{k}}^{P_{l}}} \\
\text { (Clock Hrs) }\end{array}$ & $\begin{array}{c}\left.E F T_{\exp }\right]_{R_{i}}^{P_{l} / R_{k}} \\
\text { (minutes) }\end{array}$ & $\begin{array}{l}\text { Final candidate } \\
\text { resource }\end{array}$ \\
\hline D1 & $\mathrm{R}_{1}$ & $\mathrm{P}_{4}$ & $6: 15$ & $\begin{array}{l}\mathrm{R}_{2}=12.07 \\
\mathrm{R}_{3}=17.05\end{array}$ & $\mathrm{R}_{2}$ \\
\hline D2 & $\mathrm{R}_{2}$ & $\mathrm{P}_{2}$ & $6: 13$ & $\begin{array}{l}\mathrm{R}_{1}=13.82 \\
\mathrm{R}_{3}=12.04\end{array}$ & $\mathrm{R}_{3}$ \\
\hline D3 & $\mathrm{R}_{3}$ & $\mathrm{P}_{8}$ & $6: 32$ & $\begin{array}{l}\mathrm{R}_{1}=19.51 \\
\mathrm{R}_{2}=14.60\end{array}$ & $\mathrm{R}_{2}$ \\
\hline
\end{tabular}

After the incomplete product is re-allocated, the original schedule rule is enforced once again for the remaining products. However, the active resources now always opt for the best plan and associated processing time (refer Table 1) for bidding. The corresponding schedules are shown in Fig. 6 (a) through Fig.6 (c) by Gantt charts.

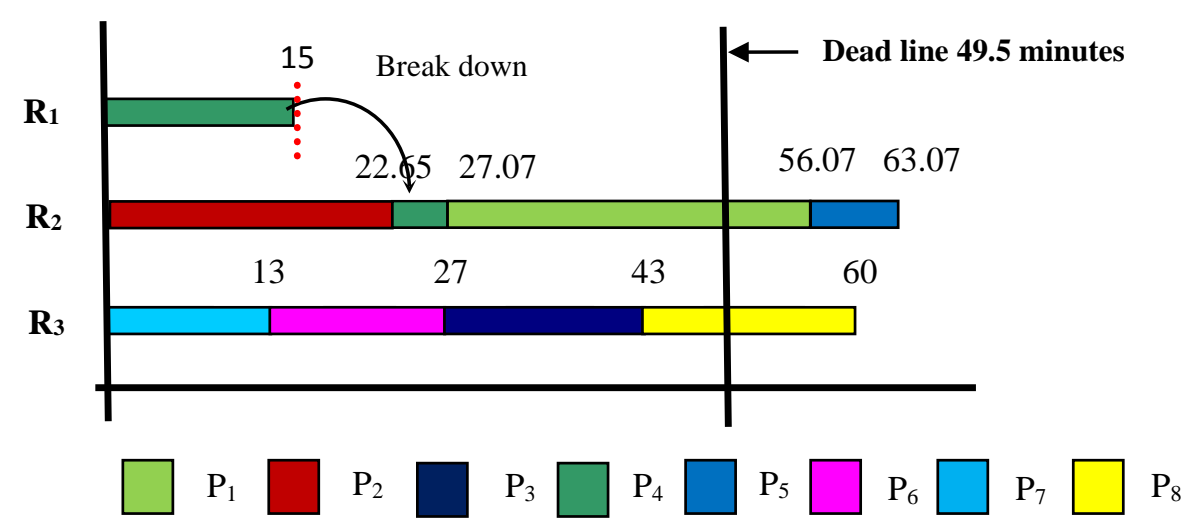

Fig. 6(a). Schedule under D1

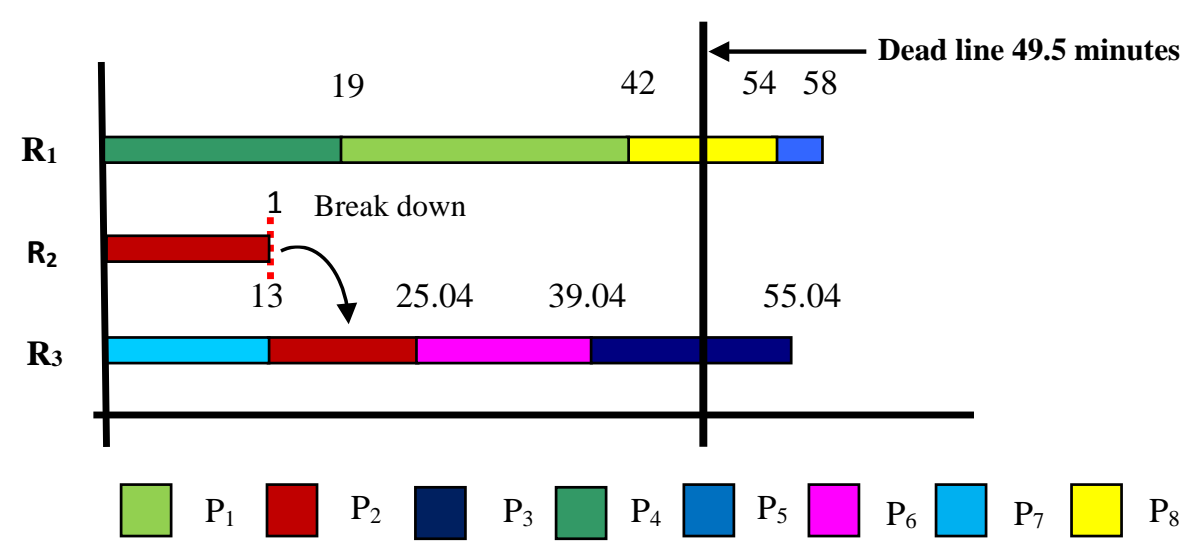

Fig.6(b). Schedule under D2 


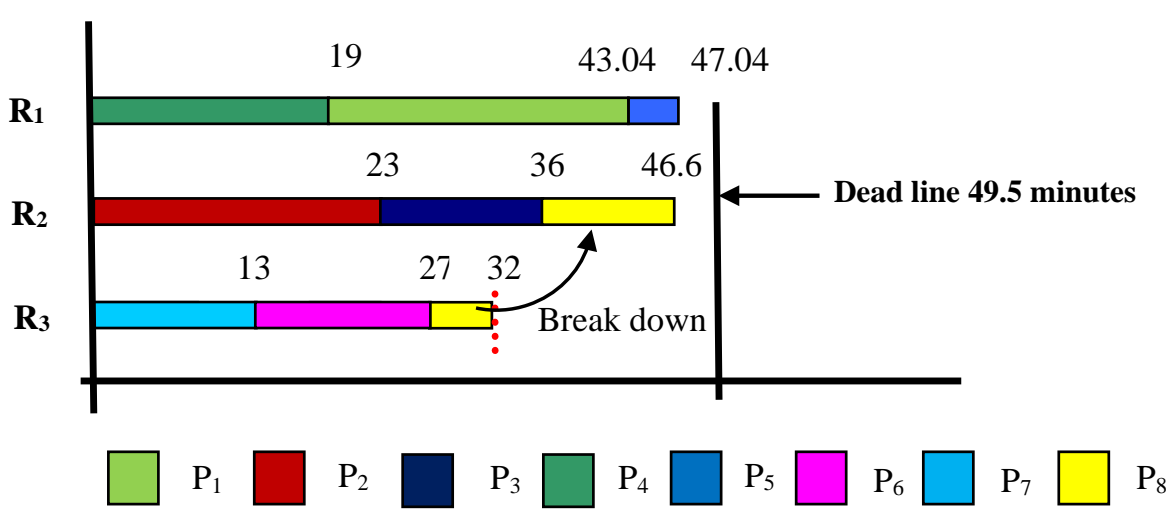

Fig. 6(c). Schedule under D3

\section{Results and Discussion}

Under normal condition $(\mathrm{N})$, the completion time becomes 44 minutes considering $\mathrm{R}_{1}$ and $\mathrm{R}_{3}$. The deadline is set at 49.5 minutes (44 minutes $+12.5 \%$ allowance). As the loading process continues, the resource load balancing automatically takes place. When any resource is confronted with malfunctioning and cannot recover immediately, the proposed metamorphic algorithm optimizes the task re-allocation. Since this procedure is merged with the original schedule rule, the load balancing among the remaining working resources is still possible as evident from Fig. 6 (a) through Fig. 6 (c). However, reduction in the number of working resources increases the total makespan which in turn causes delay. Nevertheless, in an attempt to nullify the effect of disturbance, the other resources always select the best process plan for subsequent bidding, once failure of any resource is sensed. The scheduling output under normal (N) as well as under disturbances (D1...D3) are presented in Table 3.

Table 3

Scheduling output under normal situation (N) and under disturbances (D1 - D3)

\begin{tabular}{|c|c|c|c|c|c|c|c|c|c|c|}
\hline \multirow[t]{2}{*}{ Scenario } & \multirow{2}{*}{$\begin{array}{l}\text { No. of } \\
\text { products }\end{array}$} & \multicolumn{3}{|c|}{$\begin{array}{l}\text { Engagement time of } \\
\text { Resources (minutes) }\end{array}$} & \multicolumn{3}{|c|}{ Resource utilization (\%) } & \multirow{2}{*}{ WIP } & \multirow{2}{*}{ MFT } & \multirow[t]{2}{*}{ Tardy Jobs } \\
\hline & & $\mathrm{R}_{1}$ & $\mathrm{R}_{2}$ & $\mathrm{R}_{3}$ & $\mathrm{R}_{1}$ & $\mathrm{R}_{2}$ & $\mathrm{R}_{3}$ & & & \\
\hline $\mathrm{N}$ & 08 & 44 & 43 & 44 & 88.9 & 86.8 & 88.9 & 5.70 & 31.13 & NA \\
\hline D1 & 08 & 15 & 63.07 & 60 & 30.3 & 127.4 & 121.2 & 6.78 & 38.98 & $\mathrm{P}_{1}, \mathrm{P}_{5}, \mathrm{P}_{8}$ \\
\hline D2 & 08 & 58 & 13 & 55.04 & 117.2 & 26.26 & 111.2 & 7.25 & 38.14 & $\mathrm{P}_{3}, \mathrm{P}_{5}, \mathrm{P}_{8}$ \\
\hline D3 & 08 & 47.04 & 46.6 & 32 & 95 & 94.15 & 64.6 & 5.98 & 31.33 & Nil \\
\hline
\end{tabular}

In comparison to our earlier work, it is observed that cognitive agents perform better than the ordinary agents as reflected through the reduction in MFT, although WIP remains unaltered. The reductions in MFT, however, are not very significant, since the machining time for a particular product by a resource following different process plans do not vary appreciably (refer machining time database in Table 1.). Nevertheless the merit of the work lies in the proactiveness of the cognitive agents to yield better results. The engagement time of the active resources beyond 49.5 minutes (deadline) and the \% utilization of the resources above $100 \%$ are the clear indication of delay in completion, which is accompanied by several tardy products in the scenarios D1-D2, as seen from Table 3. However, in case of $\mathrm{D} 3$ when $\mathrm{R}_{3}$ fails, the other active resources complete all the products within the stipulated time and 
the consequences of break down on WIP and MFT are not very significant, since the failure occurs at an instance when substantial progress has already been made.

\section{Conclusion}

The present paper utilizes a colony of cognitive agents to deal with machine failure while executing dynamic scheduling in an agent based holonic manufacturing system. The decision making of the cognitive agents relies on belief-desire-intention model. It is the proactiveness and self-organizing characteristics of the cognitive agents that help to provide better solutions as compared to an ordinary agent when failure of a resource tries to disrupt the work. Message of failure of any resource triggers a set of course of action as manifested through the proposed metamorphic algorithm in an attempt to find out the most suitable candidate for offering the incomplete work of the faulty resource. Under this condition, the remaining active resources always opt for the optimum process plan to gear up the processing of the subsequent tasks so as to alleviate the negative impacts of resource breakdown. The IPPS approach facilitates to improve scheduling by adopting different process plan in real-time to satisfy the changing need of the systems. The spirit of using multiple process plans is the true manifestation of the proactive behavior of the cognitive agents in an attempt to provide better solution under disturbance. The proposed cooperation and coordination mechanism is capable of handling disturbances in view of multiple resource failure also. Since the scheduling procedure is completely reactive in nature, rescheduling is required only for the affected job. Further, implicit cooperation and coordination mechanism does not call for any renegotiation and hence is free from its negative consequences. Future work in this direction would investigate the implementation opportunity of the proposed approach with necessary modifications to suit the complex industrial environments.

\section{References}

Balasubramanian, S., Brennan, R. W., \& Noorie, D. H. (2001). An architecture of metamorphic control of holonic manufacturing systems. Computers in Industry, 46, 13-31.

Bannat. A. (2011). Artificial Cognition in Production Systems. IEEE Transactions on Automation Science and Engineering, 8(1), 148-174.

Bongaerts, L., Brussel, H. V., Valckenaers, P., \& Peeters, P. (1997) Reactive scheduling in holonic manufacturing systems: architecture, dynamic model and co-operation strategy, Presented at the Advanced Summer Institute ASI 97 of the Network of Excellence on Intelligent Control and Integrated Manufacturing Systems, Budapest, Hungary, 1-10.

Brussel, H. V., Bongaerts, L., Wyns, J., Valckenaers, P., \& Ginderachter, T. V. (1999). A conceptual framework for holonic manufacturing: Identification of manufacturing holons. Journal of Manufacturing Systems, 18(1), 35-52.

Cai, N., Wang, L., \& Feng, H-Y. (2009). GA-based adaptive setup planning toward process planning and scheduling integration. International Journal of Production Research, 47(10), 2745-2766.

Cao, Y., Yang, Y., \& Yang, L. (2009). Intelligent job shop scheduling based on MAS and integrated routing wasp algorithm and scheduling wasp algorithm. Journal of Software, 4(5), 487-494.

Chan, H. K., \& Chung, S. H. (2013). Optimization approaches for distributed scheduling problems. International Journal of Production Research, 51(9), 2571-2577.

Dorigo, M., Manifesto, V., \& Colorni, A. (1996). Ant System: Optimization by a colony of cooperating agents, IEEE Transactions on Systems, Man, and Cybernetics Part B, Cybernetics, B, 26(1), 29-41.

Gao, Q., Luo, X., \& Yang, S. (2005). Stigmergic cooperation mechanism for shop floor control system. International Journal of Advanced Manufacturing Technology, 25, 743-753.

Hadeli, Valckenaers, P., Kollingbaum, M., \& Brussel, H. V. (2004). Multi-agent coordination and control using stigmergy. Computers in Industry, 53(1), 75-96.

Hirsh, A. E., \& Gordon, D. M. (2001). Distributed problem solving in social insects. Annals of Mathematics and Artificial Intelligence, 31, 199-221. 
Hsieh, F-S. (2010). Design of reconfiguration mechanism for holonic manufacturing systems based on formal models. Engineering Applications of Artificial Intelligence, 23, 1187-1199.

Huang, B., Gou, H., Liu, W., Li, Y., \& Xie, M. (2002). A framework for virtual enterprise control with the holonic manufacturing paradigm. Computers in Industry, 49, 299-310.

Jana, T. K., Bairagi, B., Paul, S., Sarkar, B., \& Saha, J. (2013). Dynamic schedule execution in an agent based holonic manufacturing system. Journal of Manufacturing Systems, 32, 801-816.

Jana, T. K., Bairagi, B., Paul, S., Sahnawaj, S., Sarkar, B. \& Saha, J. (2014). Multi-objective scheduling in an agent based holonic manufacturing system. Decision Science Letters, 3, 1-16.

Lee, S. \& Son, Y-J. (2008). Integrated human decision making model under belief-desire-intention framework for crowd simulation. Proceedings of the 2008 Winter Simulation Conference of IEEE, 886-894.

Leitao, P., \& Restivo, F. (2008). A holonic approach to dynamic manufacturing scheduling. Robotics and Computer Integrated Manufacturing, 24, 625-634.

Leitao, P. (2009). Agent-based distributed manufacturing control: A state-of-the-art survey. Engineering Applications of Artificial Intelligence, 22, 979-991.

Leitao, P. (2011). A holonic disturbance management architecture for flexible manufacturing systems. International Journal of Production Research, 49(5), 1269-1284.

Leitao, P., Barbosa, J., \& Trentesaux, D. (2012). Bio-inspired multi agent systems for reconfigurable manufacturing systems. Engineering Applications of Artificial Intelligence, 25, 934-944.

Li, X., Gao, L., Zhang, C., \& Shao, X. (2010). A review on integrated process planning and scheduling. International Journal of Manufacturing Research, 5(2), 161-180.

Li, J., Sun, S., \& Huang, Y. (2011). Adaptive hybrid ant colony optimization for solving dual resource constrained job shop scheduling problem. Journal of Software, 6(4), 584-594.

Lou, P., Ong, S. K., \& Nee, A. Y. C. (2010). Agent-based distributed scheduling for virtual job shops. International Journal of Production Research, 48(13), 3889-3910.

Meyer, G. G., Framling, K., \& Holmstrom, J. (2009). Intelligent products: A survey. Computers in Industry, 60, 137-148.

Monostori, L., Váncza, J., \& Kumara, S. R. T. (2006). Agent based systems for manufacturing. Annals of the CIRP, 55 (2), 1-24.

Nanvala, H., \& Awari, G. K. (2011). Review on use of swarm intelligence meta heuristics in scheduling of FMS. International Journal of Engineering and Technology, 3(2), 80-86.

Nejad, H. T. N., Sugimura, N., \& Iwamura, K. (2011). Agent based dynamic integrated process planning and scheduling in flexible manufacturing systems. International Journal of Production Research, 49(5), 1373-1389.

Nobre, F S., Tobias, A M. \& Walker D S. (2008). The pursuit of cognition in manufacturing organizations. Journal of Manufacturing Systems, 27, 145-157.

Ouelhadj, D., \& Petrovic, S. (2009). A survey of dynamic scheduling in manufacturing systems. Journal of Scheduling, 12(4), 417-431.

Park, H-S. \& Tran, N-H. (2012). An autonomous manufacturing system based on swarm of cognitive agents. Journal of Manufacturing Systems, 31, 337-348.

Phanden, R. K., Jain, A., \& Verma, R. (2011). Integration of process planning and scheduling: a stateof-the-art review. International Journal of Computer Integrated Manufacturing, 24(6), 517-534.

Rao, A.S. \& Georgeff, M.P. (1995). BDI agents: From theory to practice. Proceedings of the first international Conference on multi agent systems, 312-319.

Renna, P. (2011). Multi agent based scheduling in manufacturing cells in a dynamic environment. International Journal of Production Research, 49(5), 1285-1301.

Serugendo, G. D. M., Gleizes, M-P., \& Karageorgos, A. (2006). Self-organization and emergence in MAS: An overview. Informatica, 30, 45-54.

Shen, W. (2002). Distributed manufacturing scheduling using intelligent agents. IEEE Intelligent Systems, 17(1), 88-94.

Shen, W., Hao, Q., Yoon, H. J., \& Norrie, D. H. (2006a). Applications of agent-based systems in intelligent manufacturing: An updated review. Advanced Engineering Informatics, 20, 415-431. 
Shen, W., Wang, L., \& Hao, Q. (2006b). Agent based distributed manufacturing process planning and scheduling: A state-of-the-art survey. IEEE Transactions on Systems, Man, and Cybernetics - Part C: Applications and Reviews, 36(4), 563-577.

Smith, R. G. (1980). The Contract Net Protocol: high-level communication and control in a distributed problem solver. IEEE Transactions on Computer, C-29(12), 1104-1113.

Smith, R. G., \& Davis, R. (1981). Frameworks for cooperation in distributed problem solving. IEEE Transactions on Systems, Man, and Cybernetics, SMC-11(1), 61-70.

Tan, W., \& Khoshnevis, B. (2000). Integration of process planning and scheduling - a review. Journal of Intelligent Manufacturing, 11, 51-63.

Tharumarajah, A. (2003). A self-organizing view of manufacturing enterprises. Computers in Industry, 51, 185-196.

Toptal, A., \& Sabuncuoglu, I. (2010). Distributed scheduling: a review of concepts and applications. International Journal of Production Research, 48(18), 5235-5262.

Valckenaers, P., Hadeli, Germain, B. S., Verstraete, P., \& Brussel, H. V. (2007). MAS coordination and control based on stigmergy. Computers in Industry, 58(7), 621-629.

Vieira, G. E., Hermann, J. W., \& Lin, E. (2003). Rescheduling manufacturing systems: a framework of strategies, policies and methods. Journal of Scheduling, 6(1), 36-92.

Walker, S. S., Brennan, R. W., \& Norrie, D. H. (2005). Holonic job shop scheduling using a multi agent system. IEEE Intelligent Systems, 20(1), 50-57.

Wang, L., Balasubramanian, S., \& Norrie, D. H. (1998). Agent-based Intelligent Control System Design for Real-time Distributed Manufacturing Environments, Proceedings of the Agent-based Manufacturing Workshop - Autonomous Agents'98, 152-159.

Wang, L., Shen, W., \& Hao, Q. (2006). An overview of distributed process planning and its integration with scheduling. International Journal of Computer Application in Technology, 26(1/2), 3-14.

Wang, C., Ghenniwa, H., \& Shen, W. (2008). Real time distributed shop floor scheduling using an agent-based service-oriented architecture. International Journal of Production Research, 46(9), 2433-2452.

Wang, L., \& Tang, D. (2011). Optimization for manufacturing system based on pheromone. International Journal of Information Technology and Computer Science, 3, 15-21.

Wang, L., Tang, D-B., Gu, W-B., Zheng, K., Yuan, W-D., \& Tang, D-S. (2012). Pheromone based coordination for manufacturing system control. Journal of Intelligent Manufacturing, 23, 747-757.

Wong, T. N., Leung, C. W., Mak, K. L., \& Fung, R. Y. K. (2006a). Dynamic shop floor scheduling in multi agent manufacturing systems. Expert System with Applications, 31, 486-494.

Wong, T. N., Leung, C. W., Mak, K. L., Fung, R. Y. K. (2006b). Integrated process planning and scheduling/rescheduling - an agent based approach. International Journal of Production Research, 44(18-19), 3627-3655.

Wooldridge, M., \& Jennings, N. R. (1995). Intelligent agents: theory and practice. The Knowledge Engineering Review, 10(2), 115-152.

Zhao, F., Hong, Y., Yu, D., Yang, Y., Zhang, Q., \& Yi, H. (2007). A hybrid algorithm based on particle swarm optimization and simulated annealing to holon task allocation for holonic manufacturing system. International Journal of Advanced Manufacturing Technology, 32, 1021-1032.

Zhao, X. \& Son, Y. (2008). BDI-based human decision-making model in automated manufacturing systems. International Journal of Modeling and Simulation, 28, 347-356.

Zhao, F., Tang, J., Wang, J., \& Wei, C. (2011). An improved PSO algorithm with decline disturbance index, Journal of Computers, 6(4), 691-697. 